Historic, Archive Document

Do not assume content reflects current scientific knowledge, policies, or practices. 

FRED A. DANKER

\author{
40-42 Maiden Lane, Albany, N.Y. \\ PRICE LIST OF BULBS - 1926
}

EARLY SINGLE TULIPS

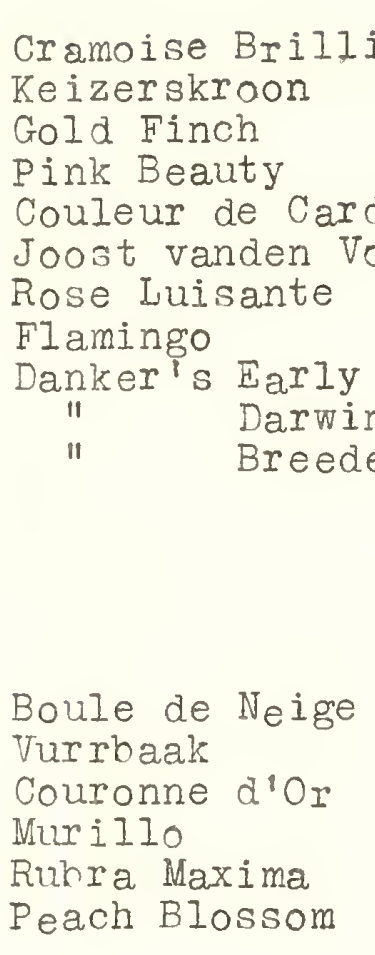

\begin{tabular}{c} 
Red \\
Red \& Yellow \\
Yellow \\
Extra Pink \\
Crimson \\
Large White \\
Best Pink \\
Red \& White \\
Iainbow Mixture \\
" \\
\multicolumn{1}{c}{$"$}
\end{tabular}

DOUBLED EARLY TULIPS

\begin{abstract}
Boule de Neige
Vurrbaak

Couronne d'Or

Runra Maxima

Peach Blossom
\end{abstract}

Toubled White

Dark Red

Orange

Pink \& White

Fine Red.

Double Pink
Joz. Hun.

.70

1.15

1.00

1.35

1.10

.90

1.00

.85

.50

.50

.70
5.00

8.00

7.00

9.50

7.75

7.00

7.00

6.00

3.50

3.50

5.00

DARWIN OR MAY FLOWER ING TULIPS

Pride of Haarlem

Baronne de la Tonnaya

Farnscombe Sanders

Inglescombe $\mathrm{Yellow}$

Clara Butt

Rev. H. Ewhanks

Bleu Amiable

Bartigan

Moonlight

Prince of the Netherland
Bright Rose

Blush Rose

Scarlet

Yellow

rink

Lilac - Pale Lav.

Violet \& Purple

Best Red

Sulphur Yellow

Soft Carmine
1.00

1.10

1.00

.75

.90

1.45
7.00

\%.75

7.00

5.50

7.00

10.00

\title{
PARROT TULIPS ASSORTED
}


HYACINTHS - IST SIZE

Grand Maitre

Queen of the Pinks

General de Wet

I'Innocence

Marconi

La Victoire

Yellow Hammer

Gertrude

Schotel

Lady Derby

La Grandesse

Marie
Blue

Pink

Pink

White

Pink

Red

Yellow

Pink

BIue

Pink

White

Deep Purple Blue

HYACINTHS - 2ND SIZE

\begin{tabular}{|c|c|c|}
\hline Each & Noz. & Hun. \\
\hline .25 & $3_{i}, 00$ & $22_{11} .50$ \\
\hline 11 & $"$ & $"$ \\
\hline$\|$ & " & 11 \\
\hline " & $"$ & 11 \\
\hline$"$ & " & " \\
\hline " & " & 11 \\
\hline " & " & $\|$ \\
\hline 11 & $"$ & $\|$ \\
\hline " & $"$ & 11 \\
\hline 11 & $"$ & $v$ \\
\hline$n$ & $"$ & " \\
\hline
\end{tabular}

$\begin{array}{ll}\text { Grand Maitre } & \text { Blue } \\ \text { Queen of the Pinks } & \text { Pink } \\ \text { General de Wet } & \text { Pink } \\ \text { I Innocence } & \text { White } \\ \text { Marconi } & \text { Pink } \\ \text { La Victoire } & \text { Red } \\ \text { Yeliow Hammer } & \text { Yelluw } \\ \text { Gertrude } & \text { Pink } \\ \text { Schotel } & \text { BIue } \\ \text { Lady Derby } & \text { Pink } \\ \text { La Grandesse } & \text { White } \\ \text { Marie } & \text { Deep Purple Blue }\end{array}$

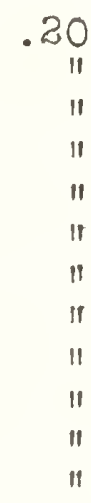

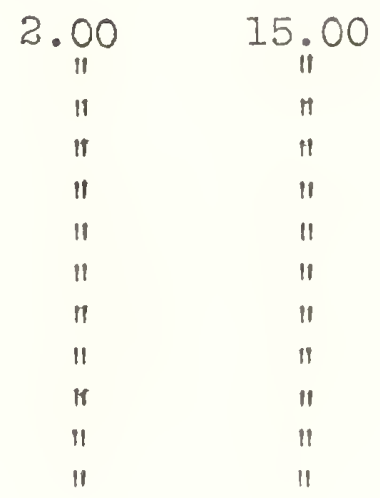

\section{DAFFODILS FOR GARDEN AND HOUSE CULTURE}

\section{Poeticus Ornatus}

(Or Poet's Narcissus) White

Emperior

Victorie

Sir Watkins

King Alfred

Golden Spur
Iarge Yellow

Yollow \& White

Yellow

Giant Yellow

$\begin{array}{rr}.95 & 7.00 \\ 1.75 & 13.00 \\ 1.75 & 13.00 \\ 1.75 & 13.00 \\ 3.00 & 23.00 \\ 1.75 & 13.00\end{array}$


